\title{
Response Surface Optimization For Clarification Of White Pitaya Juice Using A Commercial Enzyme
}

\begin{abstract}
Raw white pitaya (Hylocereus undatus) juice is very cloudy and viscous. These physical properties lead to the use of enzymatic treatment to clarify the juice. The aim of this study was to obtain the optimum process conditions for enzymatic clarification using response surface methodology (RSM). White pitaya juice was treated with pectinase enzyme (Pectinex Ultra SP-L) at different maceration times (20-100 $\mathrm{min})$, reaction temperatures (30-50C) and enzyme concentrations $(0.01-0.1 \%)$. These three variables were used as independent variables whose effects on yield, viscosity, clarity and color were evaluated. From the RSM analysis, the recommended enzymatic treatment condition from the study was found at $0.06 \%$ enzyme concentration at $49 \mathrm{C}$ for $40 \mathrm{~min}$.
\end{abstract}

